\title{
Stromal interaction essential for vascular endothelial growth factor A-induced tumour growth via transforming growth factor- $\beta$
} signalling

\section{AC Weidenaar', A ter Elst', KR Kampen', TGJ Meeuwsen-de Boer', HJM de Jonge', FJG Scherpen', WFA den Dunnen', WA Kamps' and ESJM de Bont*,}

'Division of Pediatric Oncology/Hematology, Department of Pediatrics, Beatrix Children's Hospital, University Medical Center Groningen, University of Groningen, PO Box 30.00 I, Groningen 9700 RB, The Netherlands; ${ }^{2}$ Division of Pathology, Department of Pathology and Medical Biology, University Medical Center Groningen, University of Groningen, Groningen, The Netherlands

BACKGROUND: High vascular endothelial growth factor (VEGFA) levels at the time of diagnosis confer a worse prognosis to multiple malignancies. Our aim was to investigate the role of VEGFA in promoting tumour growth through interaction with its environment. METHODS: HL-60 cells were transduced with VEGFAI65 or control vector using retroviral constructs. Control cells $(n=7)$ or VEGFAI 65 cells $(n=7)$ were subcutaneously injected into NOD/SCID mice. Immunohistochemistry of markers for angiogenesis (CD3I) and cell proliferation (Ki67) and gene expression profiling of tumours were performed. Paracrine effects were investigated by mouse-specific cytokine arrays.

RESULTS: In vivo we observed a twofold increase in tumour weight when VEGFAI 65 was overexpressed $(P=0.00 \mathrm{I})$, combined with increased angiogenesis $(P=0.002)$ and enhanced tumour cell proliferation $(P=0.00 \mathrm{I})$. Gene expression profiling revealed human genes involved in TGF- $\beta$ signalling differentially expressed between both tumour groups, that is, TGFBR2 and SMAD5 were lower expressed whereas the inhibitory SMAD7 was higher expressed with VEGFAI65. An increased expression of mouse-derived cytokines IFNG and interleukin 7 was found in VEGFAI 65 tumours, both described to induce SMAD7 expression.

CONCLUSION: These results suggest a role for VEGFA-driven tumour growth by TGF- $\beta$ signalling inhibition via paracrine mechanisms in vivo, and underscore the importance of stromal interaction in the VEGFA-induced phenotype.

British Journal of Cancer (201I) I 05, 1856- 1863. doi: I0.1038/bjc.201 I.460 www.bjcancer.com

Published online I November 201 |

(c) 20II Cancer Research UK

Keywords: VEGFA; tumourigenesis; stroma; angiogenesis; TGF- $\beta$

The level of vascular endothelial growth factor (VEGFA) at time of diagnosis is an independent prognostic factor for treatment outcome in multiple malignancies, including (paediatric) acute myeloid leukaemia (AML) (Aguayo et al, 1999; de Bont et al, 2002). As a result, targeting VEGFA has been a subject of extensive research during the last decades.

In general, tumour growth is supported by angiogenesis, the formation of new blood vessels. An increase in microvessel density (MVD) is seen in many malignancies. In AML, enhanced bone marrow angiogenesis at diagnosis was shown, recovering to normal levels when CR was achieved (Padro et al, 2000; de Bont et al, 2001). Moreover, a correlation was found between the degree of VEGFA expression and the increase in bone marrow vascularisation at AML presentation (de Bont et al, 2001).

Co-expression of VEGFA and its tyrosine kinase receptors VEGFR1 and VEGFR2 has been reported previously in AML. Upon binding of its ligand, both receptors can activate several downstream pathways such as PI3-kinase and MAPK signalling, inducing cell proliferation and cell survival (Lewis et al, 1998;

*Correspondence: Dr ESJM de Bont; E-mail: e.s.j.m.de.bont@bkk.umcg.nl Received 4 October 201 I; accepted 6 October 201 I; published online I November 201 I
Bellamy et al, 1999; Grandage et al, 2005). Apart from autocrine effects, secreted VEGFA stimulates endothelial and stromal cells that may drive leukaemic cell proliferation via paracrine effects (Fiedler et al, 1997; Lee et al, 2007).

Although a role for VEGFA in malignant progression was implicated, the exact mechanism by how increased VEGFA levels influence tumour growth is still not completely understood. In this study a model was generated to investigate the effect of VEGFA in tumour progression. Using a human AML cell line transduced with VEGFA165 or empty vector (negative control), we were able to show that in vitro overexpression of VEGFA165 did not influence cell growth patterns and drug resistance, whereas in vivo VEGFA165 overexpression enhanced tumour outgrowth and induced the expression of host-derived cytokines. Our study suggests that VEGFA165-promoted tumour growth was mediated by a paracrineinduced inhibition of the TGF- $\beta$ signalling pathway, and underlines the importance of targeting the tumour microenvironment.

\section{MATERIALS AND METHODS}

\section{Cell line culture and transduction}

The human AML cell line HL-60 was obtained from the ATCC (Manassas, VA, USA) in 2006. Cells have last been tested and 
authenticated by flow-cytometry analysis in 2009. HL-60 cells were cultured in RPMI-1640 medium supplemented with $1 \%$ penicillin/ streptomycin and $10 \%$ fetal bovine serum (Hyclone, Logan, UT, USA). Retroviral supernatants were generated by cotransfection of $2 \mu \mathrm{g}$ reporter constructs pMSCV-iGFP-VEGFA165 or pMSCV-iGFP (empty vector, negative control) and $2 \mu \mathrm{g}$ packaging plasmid pCLampho into 293T cells using FuGENE HD transfection reagent (Roche, Almere, the Netherlands). HL-60 cells $\left(5 \times 10^{4}\right)$ were incubated with retroviral supernatants, which were filtered through $0.45-\mu \mathrm{m}$ pore size syringe-mounted filters. Incubation was supplemented with $8 \mu \mathrm{g} \mathrm{ml}^{-1}$ polybrene. This procedure was repeated for 2 consecutive days after which stably transduced cells were expanded. Transduction efficiency was measured by FACS analysis, which demonstrated an efficiency of $25 \%$ for cells transduced with the empty vector and $10 \%$ for cells transduced with VEGFA165. Cells were sorted on a MoFlo (DAKO Cytomation, AS, Glostrup, Denmark); after sorting the percentage of transduced cells was $90 \%$ for the empty vector (control cells) and $67 \%$ for the VEGFA165 vector (VEGFA165 cells). Cells were cultured overnight under described serum conditions before use.

\section{Animal study}

NOD/SCID mice (kindly provided by Dr LD Shultz, The Jackson Laboratory, Bar Harbor, ME, USA) were bred and maintained at the Central Animal Facility, University of Groningen, Groningen, the Netherlands. Animals were kept under laminar flow conditions during the experiment. Our model was generated by subcutaneously injecting $10 \times 10^{6}$ VEGFA165 cells (VEGFA165 tumour) or control cells (control tumour) into the right flank of sublethally irradiated (2 Gy) 6-8-week-old NOD/SCID mice $(n=14)$. Mice were monitored for 15 days, during which tumour growth was assessed periodically. Tumour volumes were determined by external measurement according to the equation $\left(V=L \times W^{2}\right) \times 0.5$, where $V=$ volume, $L=$ length and $W=$ width (Tsujii et al, 1998). Mice were killed using cervical dislocation at day 15 and tumours were harvested. Tumours were split and either snap-frozen or fixed in formalin and embedded in paraffin for immunohistochemistry or other analyses. All procedures involving animals were performed in accordance with local ethical animal laws and policies.

\section{RNA extraction and real-time PCR}

Total RNA from frozen tumour material was extracted with NucleoSpin RNA II kit according to the manufacturer's protocol (Macherey-Nagel, Duren, Germany). Complementary DNA was prepared at $37^{\circ} \mathrm{C}$ for at least $1 \mathrm{~h}$ in $20 \mu \mathrm{l}$ reaction mixture containing $2 \mu \mathrm{g}$ of total RNA, random hexamers (Pfizer, Capelle a/d IJssel, the Netherlands), $5 \times$ first strand buffer, RNAsin and reverse transcriptase (Gibco BRL, Grand Island, NY, USA). Real-time PCR was performed using iQ SYBR green supermix (Bio-Rad, Hercules, CA, USA). All PCR reactions and data analysis were performed on the iCycler iQ Real-Time Detection System (Bio-Rad). Expression of genes of interest was standardised for expression of hRPL22 or $\mathrm{m} \beta$-actin (arbitrary units, AU). Humanspecific (h) and mouse-specific (m) primers for the reverse transcriptase PCR are listed in Supplementary Table S1. The primers were checked in NCBI blasts and did not occur in mouse genome or transcriptome. In addition, no PCR product could be detected in mouse tissue with these primers.

\section{ELISA and functional assay of VEGFA}

Secretion of VEGFA was detected in the supernatant using commercially available ELISAs (Quantikine immunoassays, R\&D Systems, Abingdon, UK) following the manufacturer's instructions. The functionality of secreted VEGFA from transduced cells was detected by adding its supernatant to endothelial cells and quantify expression of VEGFA-specific genes EGR3, NUR77 and NOR1 in endothelial cells with real-time PCR, described in detail by Liu et al (2003).

\section{Flow cytometry analysis}

For BrdU incorporation, a measure of DNA-synthesis, $0.5 \times 10^{6}$ cells were incubated for 2,4 or $8 \mathrm{~h}$ with $0 \%$ or $10 \%$ fetal calf serum in presence of $1 \mu \mathrm{M}$ demecolcine (Sigma, St Louis, MO, USA) and $10 \mu \mathrm{M}$ BrdU (BD, Alphen a/d Rijn, the Netherlands), and prepared according to the BdrU-assay protocol following the manufacturer's instructions (BD); cells were neutralised for acids in $0.5 \mathrm{M}$ EDTA, secondary antibody: phycoerythrin-conjugated anti-mouse (DAKO, AS, Glostrup, Denmark). Measurements with FACScalibur LSR-II (BD), data were analysed using FlowJo.

\section{Cellular drug resistance measurement using total cell kill assay}

A total cell kill assay was performed on VEGFA165 cells and control cells using Amsacrine $\left(0.05-2 \mu \mathrm{g} \mathrm{ml}^{-1}\right)$ in different concentrations in quadruplicate following former publications (Weidenaar et al, 2008). Optical density in the total cell kill assay is linearly related to the number of viable cells.

\section{Microarray analysis}

RNA from 14 tumour samples was analysed using Affymetrix Human Genome U133 Plus 2.0 GeneChip (Affymetrix, Santa Clara, CA, USA). RNA quality control, cDNA labelling, microarray hybridisation, scanning, data extraction and data normalisation were performed by ServiceXS, Leiden, the Netherlands. Differentially expressed genes were identified for tumours using multivariate permutation test in Biometric Research Branch ArrayTools (BRB ArrayTools). BRB ArrayTools has been developed by the Biometric Research Branch of the US National Cancer Institute (http://linus.nci.nih.gov/BRB-ArrayTools.html). A total of 1000 permutations were completed to identify the list of probe sets. Differentially expressed probe sets were identified using a twosample $t$-test, threshold $P<0.001$ and false discovery rate (FDR) of $<0.25$ was used. Gene ontology (GO) categories and KEGG pathways were determined using DAVID (Database for Annotation, Visualization and Integrated Discovery, Bioinformatics Resources 6.7, National Institute of Allergy and Infectious Diseases (NIAID), NIH, http:// david.abcc.ncifcrf.gov/knowledgebase). Probe sets with a significance level of 0.001 and FDR $<0.25$ were used for GO analysis. Gene set expression comparisons were performed with univariate two sample $t$-test at a significance level of $P<0.05$, followed by 200 permutations. Affymetrix array results were validated using quantitative (Q)RT-PCR with human-specific primers for 11 genes of interest (Supplementary Figure S1).

\section{Immunohistochemical analysis for vessel density and proliferation}

Frozen tumour samples were cut into $4-\mu \mathrm{m}$ sections and studied for vessel density by staining with CD31 (platelet endothelial cell adhesion molecule (PECAM)-1) as well as for proliferation by staining with Ki67. Sections were blocked for endogene peroxidase with $0.25 \% \mathrm{H}_{2} \mathrm{O}_{2}$ and incubated with mouse-specific CD31 (BD). Subsequently, sections were incubated with secondary antibody (swine) anti-rat biotin (DAKO), amplified with (biotin) streptavidin ABComplex/HRPO (DAKO) and detected by 3-amino-9ethylcarbazole (Sigma). After that slides were coloured with haematoxylin. Negative controls were produced using non-specific IgG as primary antibody. Sections for Ki67 were treated similarly except for the antibodies: primary monoclonal mouse anti-human Ki67 antibody (DAKO) and secondary antibody (rabbit) antimouse biotin (DAKO). Vessel density was assessed using light 
microscopy at $\times 50$ magnification in areas of the slide containing the highest numbers of microvessels representing most intense microvasculature (hotspots). After the hotspots were identified, total number of vessels per selected image was counted at $\times 400$ magnification. At least four hotspots were counted for each section. Proliferation was evaluated by the percentage of Ki67 positive tumour cells in four hotspot areas (selected at $\times 50$ magnification): the selected image was divided into four areas, and the percentage of Ki67-positive tumour cells was estimated for all four areas at $\times 400$ magnification. The mean of the four estimates was considered the count of that hotspot. Stainings were evaluated by two investigators who had no knowledge of tumour characteristics. Variability between investigators for vessel count was $\rho=0.993$ and for proliferation count was $\rho=0.996$. The mean of the two independent counts was considered to be the final measurement for each counting field and hotspot.

\section{Cytokine array}

In VEGFA165 $(n=4)$ and control $(n=4)$ tumours 40 mousespecific cytokine levels were determined by using proteome profiler mouse cytokine array panel A kit (R\&D Systems) according to manufacturer's instructions $(400 \mu \mathrm{g}$ protein of each sample was added). Spot densities were quantified with Scanalyze software (http://rana.lbl.gov/EisenSoftware.htm) and exported to Microsoft Excel. Spot densities were corrected for individual background to diminish interarray variances. To assess the mouse specificity of the cytokine array, protein extracts of the human HL-60 cell line and of the mouse MS5 cell line were applied to the cytokine array. As expected, a strong signal was found for the MS5 mouse cells, and no signal could be detected for the HL-60 control cells (Supplementary Figure S2).

\section{Statistical analysis}

Wilcoxon signed-rank test was used to compare the growth of both cell lines. Tumour volumes between VEGFA165 tumours and control tumours were determined using the Student's $t$-test. Differences between tumour volumes at indicated time points were calculated using the Student's paired $t$-test. Correlation between expression of VEGFA165, tumour volume, tumour weight, percentage of cell proliferation and MVD were calculated using Spearman's correlation. Mann-Whitney $U$-test was used for comparison of vessel and proliferation counts, QRT-PCR as well as mouse-specific cytokines differentially expressed within VEGFA165 tumours and control tumours. $P<0.05$ was considered significant.

\section{RESULTS}

\section{Functional VEGFA165 overexpression in HL-60 cells}

We transduced HL-60 cells with VEGFA165 (retroviral vectors schematically depicted in Figure 1A). VEGFA165 mRNA expression and secreted protein levels were 3-4-fold upregulated in VEGFA165 cells compared with control cells (Figure 1B and C). To test the functionality of the produced VEGFA165, conditioned medium of VEGFA165-transduced cells or control cells was added to endothelial cells (HUVECs) and the mRNA expression of a VEGFA responsive gene EGR3 was measured. A fivefold upregulation of EGR3 was detected in HUVEC cells after incubation with conditioned medium of VEGFA165-transduced cells compared with conditioned medium of control cells (Figure 1D). FACS analysis showed KDR expression in VEGFA165-transduced cells similar to control cells. In vitro cell proliferation and cell growth was similar in both cell lines (Figures $1 \mathrm{E}$ and F) and no difference in drug resistance was observed between VEGFA165 cells and control cells (data not shown).

\section{Overexpression of VEGFA165 results in increased angiogenesis and cell proliferation in a s.c. xenograft mouse model}

To investigate the role of VEGFA165 overexpression in vivo, we subcutaneously inoculated VEGFA165 cells or control cells in mice. Tumour volume of VEGFA165 tumours increased more rapidly than control tumours, with a significant difference at day 13 and $15(P<0.05$ and $P<0.01$ respectively, Figure $2 \mathrm{~A})$. When tumour-bearing mice were killed at day 15 tumour weight of VEGFA165 tumours (median weight $995 \mathrm{mg}$, range 670-1344) was significantly $(P=0.001)$ increased compared with the control tumours (median weight $464 \mathrm{mg}$, range 413-646) (Figure 2B). MVD in VEGFA165 tumours (median MVD 64.9 vessels per hpf, range 54.4-88.6) was significantly $(P=0.002)$ enhanced compared with control tumours (median MVD 46.1 vessels per hpf, range $39.9-56.5)$, and a significant $(P=0.001)$ increase in tumour cell proliferation fraction in VEGFA165 tumours was found (median: $76.3 \%$, range 66.3-91.6) compared with control tumours (median: $50.3 \%$, range 35.4-65.6) (Figure 2C-H). Interestingly, tumour weight was significantly correlated to MVD $(\rho=0.566, P=0.044)$ and percentage of proliferating cells $(\rho=0.722, P=0.004)$. In contrast to in vitro results, in vivo a clear phenotype could be appreciated as increased angiogenesis and tumour cell proliferation was evident.

\section{Distinct gene expression profiles related to VEGFA165 overexpression in vivo}

To obtain a more detailed understanding of the phenotype within the tumour cells overexpressing VEGFA165, mRNA of VEGFA165 tumours and control tumours was isolated, amplified and hybridised to Affymetrix human U133 Plus 2.0 GeneChips. Class comparison analysis revealed 761 probe sets to be differentially expressed between the two tumour groups; 242 probe sets were higher expressed in VEGFA165 tumours whereas 519 probe sets were lower expressed in these tumours (complete list shown in Supplementary Table S2). As expected, VEGFA was significantly higher expressed in the VEGFA165 tumours compared with control tumours $\left(P<1 \times 10^{-5}\right)$.

Subsequently, GO analysis of the upregulated probe sets in VEGFA165 tumours revealed enrichment for the process of 'angiogenesis' (GO:0001525, $P=0.03$ ) in accordance with the immunohistochemical staining for MVD. The GO term 'cell death' (GO: $0008219, P=0.02$ ) was enriched in the downregulated probe sets of the VEGFA165 tumours, underscoring the observed increase in tumour growth and cell proliferation (complete list shown in Supplementary Table S3).

The list of up and downregulated genes in VEGFA165 tumours include known genes involved in neoplasia (e.g. S100A8 involved in leukaemia; S100A9 in prostate carcinoma; BCL9, CCND1 and CTNNB1) (Hermani et al, 2006; Mani et al, 2009; Nicolas et al, 2011; Siapati et al, 2011). In addition, the cytokine receptors $I L 4 R$ and IL7R were found to be higher expressed in VEGFA165 tumours implicating the possibility of paracrine effects.

Notable, genes involved in TGF- $\beta$ signalling (SOS1, SOS2, SMAD5, LTBP3, TGFBR2, IRF7 and CREBZF) were found to be significantly $(P<0.01)$ downregulated in the VEGFA165 tumours. In addition, SMAD7, a negative modulator of TGF- $\beta$ signalling, was found to be upregulated in VEGFA165 tumours. Three other genes involved in the TGF- $\beta$ signalling pathway were also found to be significantly $(P<0.05)$ differentially expressed (SMAD3, IRF7, SMURF2).

To validate these results QRT-PCR with human-specific primers was performed for 11 genes of interest ( $h V E G F A, h C R E B Z F$, hLTBP3, hIL4R, hIL7R, hSOS1, hSOS2, hSMAD3, hSMAD7, hSMURF2 and hTGFBR2). Differential expression of these 11 genes could be confirmed using QRT-PCR (Supplementary 
A

\begin{tabular}{|c|c|c|c|c|}
\hline $5^{\prime}$ LTR & - & IRES & EGFP & 3'LTR $^{\prime \prime}$ \\
\hline $5^{\prime}$ LTR & VEGFA165 & IRES & EGFP & $3^{\prime} \mathrm{LTR}$ \\
\hline
\end{tabular}
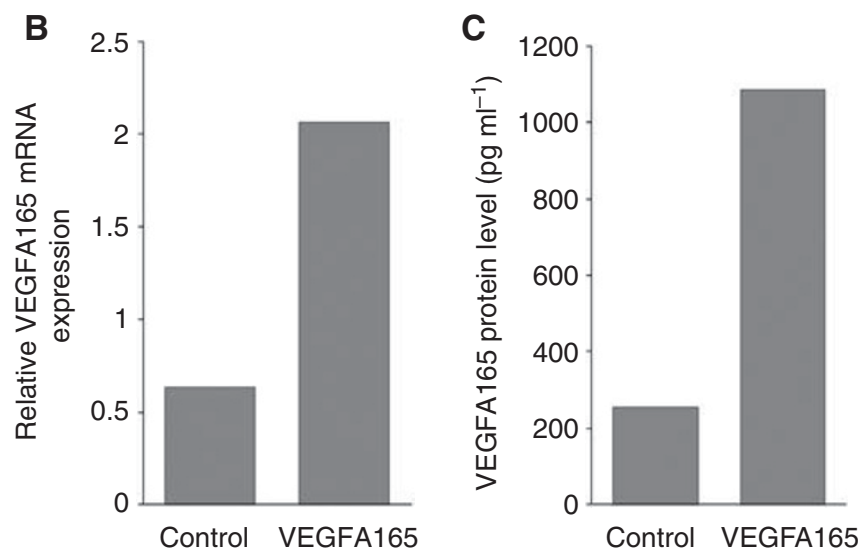

$\mathbf{F}$

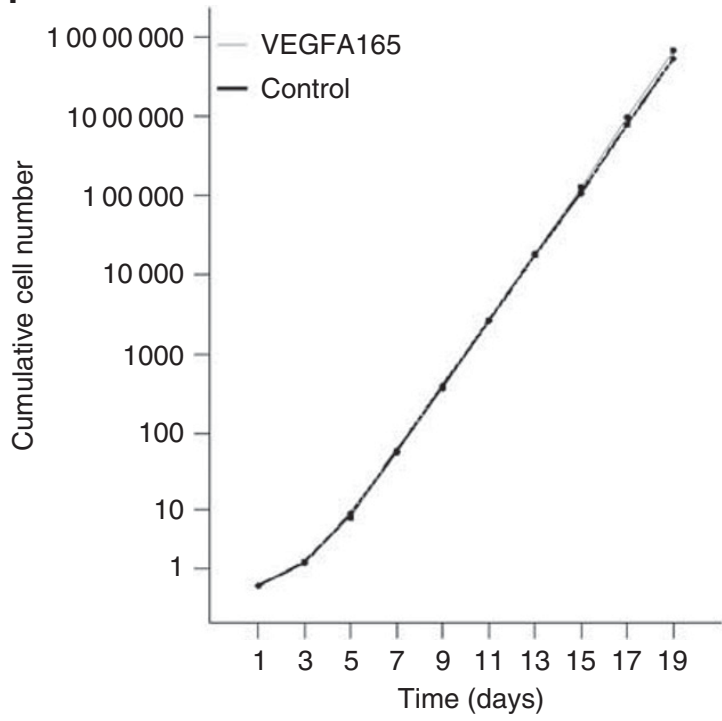

D

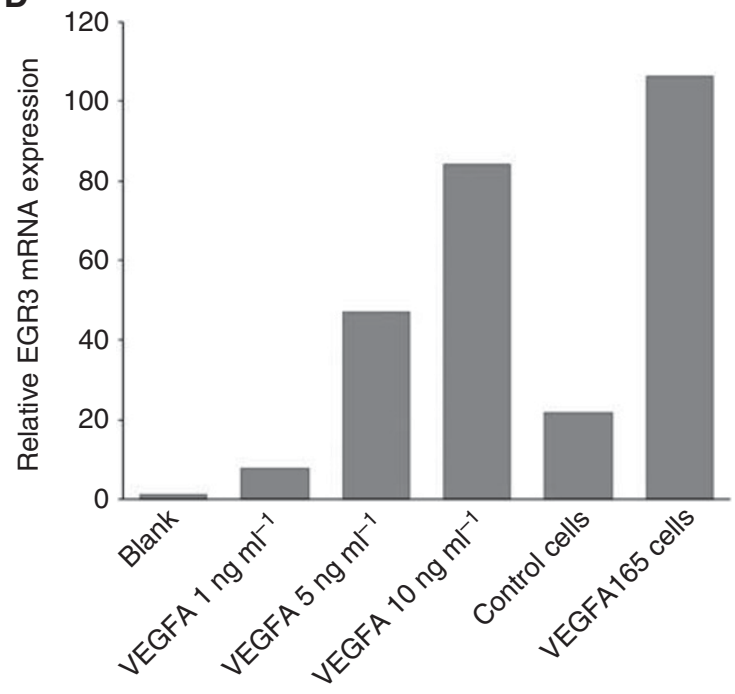

E
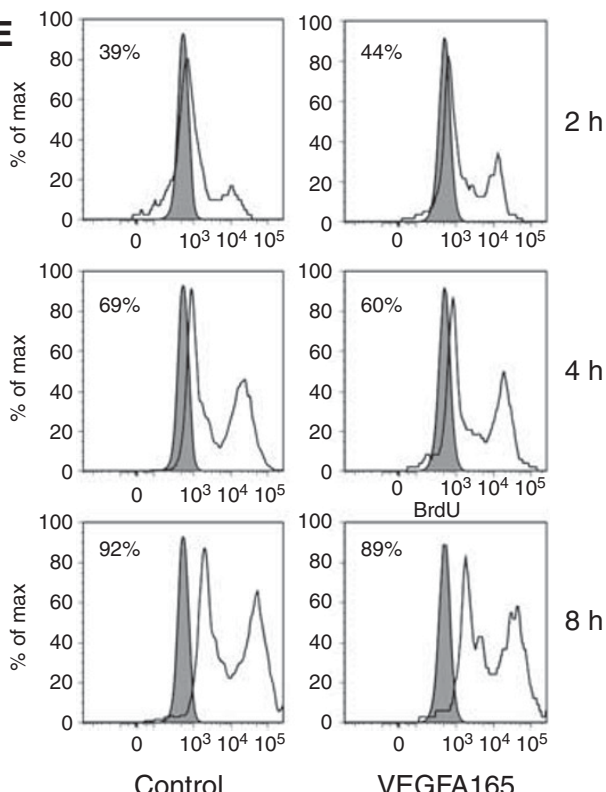

Figure I Effect of VEGFA 65 overexpression in vitro. (A) Schematic representation of the retroviral constructs that were used in this study. (B) Relative mRNA expression of VEGFA 65 in control cells compared with VEGFAI65 cells. (C) Protein expression of VEGFAI 65 in control cells compared with

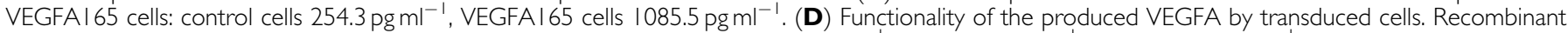

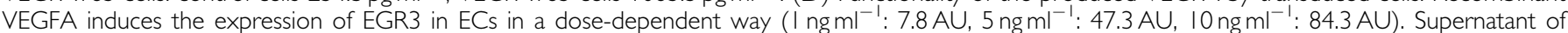
transduced cells contained functional VEGFA, indicated by the expression of EGR3. The VEGFAI 65 cells showed a higher expression of EGR3 than the control cells, demonstrating that VEGFAI 65 cells produce more functional VEGFA (control cells: 22.0 AU; VEGFA I 65 cells: I06.5 AU). Similar graphs were seen when investigating the expression of NUR77 and NORI. (E) BdrU incorporation of control cells and VEGFAI65 cells cultured in serum-free conditions. After 2, 4 and $8 \mathrm{~h}$ cells were harvested and the percentage of cells that incorporated BrdU was analysed by flow cytometry. Percentage of BrdUincorporating cells: after $2 \mathrm{~h} 39 \%$ vs 44\%; after $4 \mathrm{~h} 69 \%$ vs 60\%; after 8 h $92 \%$ vs $89 \%$. Background staining in the absence of BrdU was I\%. Similar graphs were seen when cells were cultured with $10 \%$ fetal calf serum. (F) No significant difference in cell growth of VEGFAI 65 cells compared with control cells was seen when cells were cultured with $10 \%$ fetal calf serum $(P=0.169$, Wilcoxon signed-rank test).

Figure S1). Therefore, we conclude that the genes involved in the TGF- $\beta$ signalling pathway are derived from human cells within the tumours.

\section{Paracrine mechanisms have important roles in VEGFA165 tumours}

We hypothesised that the in vivo growth benefit of high VEGFA165 levels occurs via interaction with its (micro)environment. In the VEGFA165 tumours mouse-specific VEGFR2 mRNA was twofold upregulated $(P=0.001$, Supplementary Figure $S 1)$, the main receptor for VEGFA signalling. Using mouse-specific cytokine arrays (tested for mouse specificity, Supplementary Figure S2), we assessed whether the increased tumour growth would be reflected in altered mouse-derived cytokine expression. Five mouse-specific cytokines were significantly $(P=0.03)$ higher expressed in VEGFA165 tumours compared with control tumours: interleukin 5 (IL5), IL7, chemokine (C-X-C motif) ligand 9 (CXCL9, MIG), colony-stimulating factor 1 (macrophage) (MCSF) and interferon $\gamma$ (IFNG) (Figure 3A). Tissue metallopeptidase inhibitor 1 (TIMP1) 

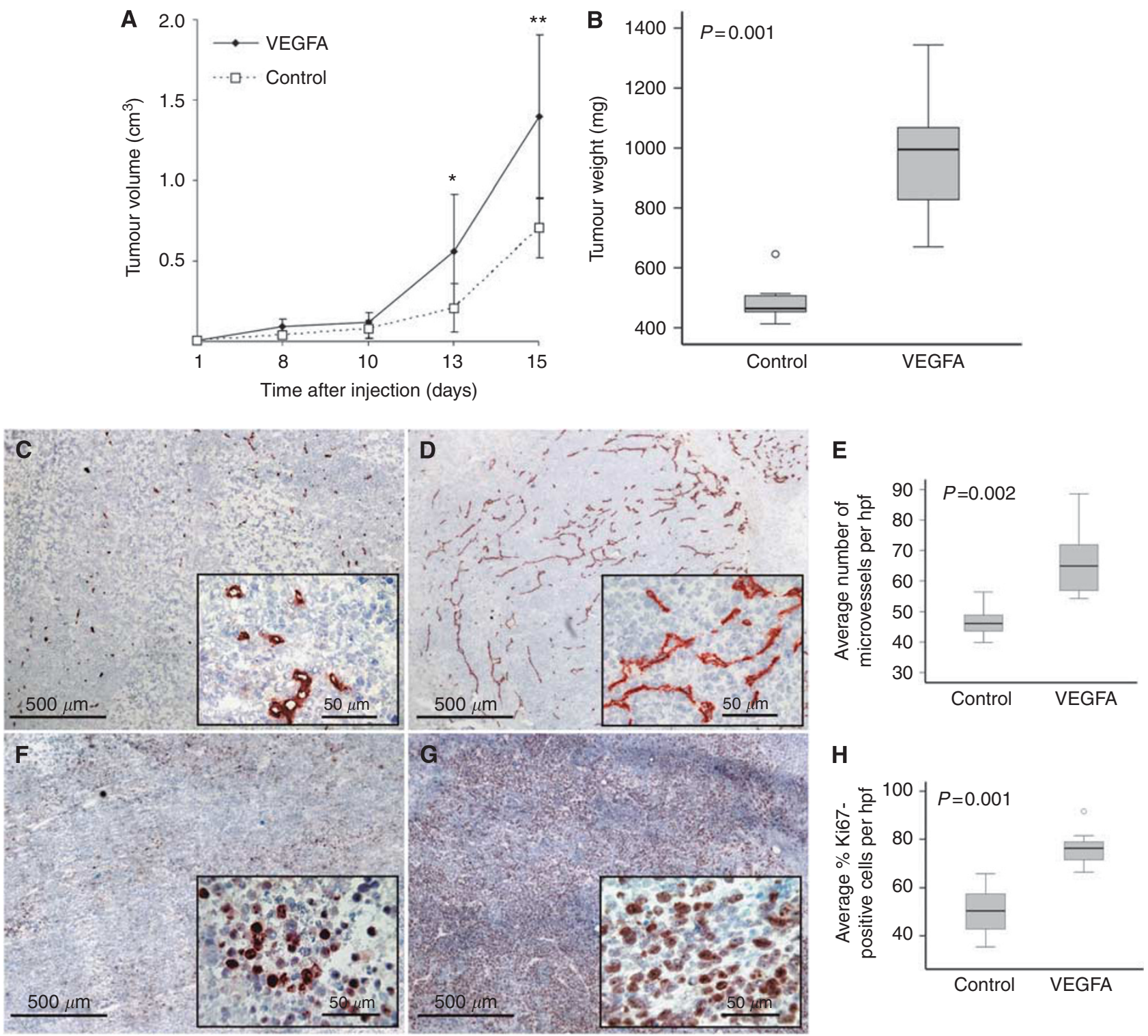

Figure 2 Effect of VEGFAI 65 overexpression in vivo. (A) Tumour volumes were assessed periodically. At day I3 and day I5 a significant difference was seen. Values expressed are the means \pm s.e.m. of seven xenografts per group. $* P<0.05$, $* * P<0.01$. (B) Tumour weight of tumours derived from $V E G F A I 65$ cells was significantly increased compared with tumour weight of control tumours at day I5 $(P=0.00$ I). Median weight of VEGFAI 65 tumours: 995 mg (range 670-1344), control tumours: $464 \mathrm{mg}$ (range 4I3-646). (C) CD3I staining of a VEGFAI65 tumour and (D) a control tumour. (E) VEGFAI65 tumours showed a significantly $(P=0.002)$ higher MVD compared with the control tumours. Median MVD control tumours: 46.I per field (range 39.956.5); median MVD VEGFAI 65 tumours: 64.9 vessels per field (range 54.4-88.6). (F) Ki67 staining of a VEGFAI 65 tumour and (G) control tumour. (H) A significantly $(P=0.00 \mathrm{I})$ higher percentage of proliferating cells was found in the VEGFAI65 tumours compared with the control tumours. Median percentage of proliferating cells control tumours: 50.3\% (range 35.4-65.6); median percentage of proliferating cells VEGFAI65 tumours: $76.3 \%$ (range $66.3-91.6)$. A representative photograph from each group is shown (original magnification $\times 50$, with inset $\times 400$ magnification).

was found to be significantly $(P=0.03)$ lower expressed in VEGFA165 tumours compared with control tumours (Figure 3B). Together these data show that stroma-derived (i.e. mouse-derived) cytokines are regulated in tumours overexpressing VEGFA, suggesting that VEGFA exerts its effect on tumour growth via a paracrine loop.

\section{DISCUSSION}

In this study we demonstrated that VEGFA165 overexpression significantly enhanced tumour progression in vivo, accompanied by a higher percentage of proliferating tumour cells and increased angiogenesis. We hypothesise that the enhanced tumour cell proliferation is accompanied by inhibition of the TGF- $\beta$ signalling pathway in the (human) tumour cells via various stromal-derived mouse cytokines such as IL7 and IFNG (Figure 4).

High VEGFA levels predict a poor prognosis in multiple malignancies, including haematological malignancies. In our study, in vitro induction of high VEGFA165 levels did not alter the proliferative status of VEGFA165 cells compared with control cells despite the fact that HL-60 cells express VEGFR2 and the possibility of an autocrine loop has been described (Weidenaar et al, 2008). In contrast, overexpression of VEGFA165 in vivo resulted in an increased tumour growth and higher levels of proliferating cells. 

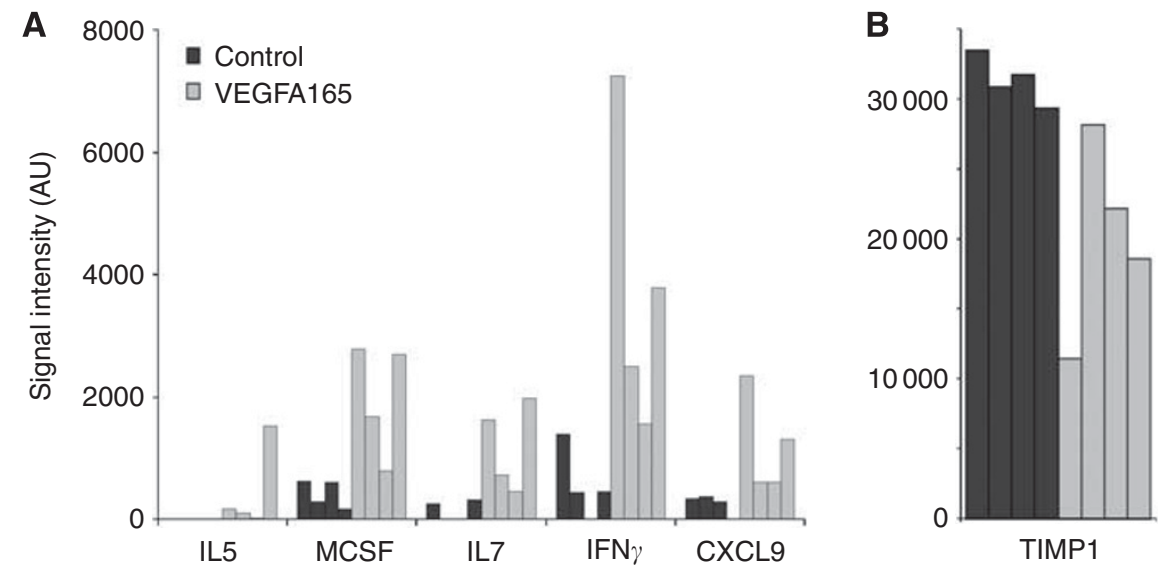

Figure 3 Expression of mouse-specific cytokines. Mouse-specific cytokines significantly $(P=0.03)$ differentially expressed between the VEGFAI65 tumours $(n=4)$ and control tumours $(n=4)$, showing five cytokines higher expressed $(\mathbf{A})$ and one cytokine lower expressed in the VEGFAI65 overexpressing tumours $(\mathbf{B})$.

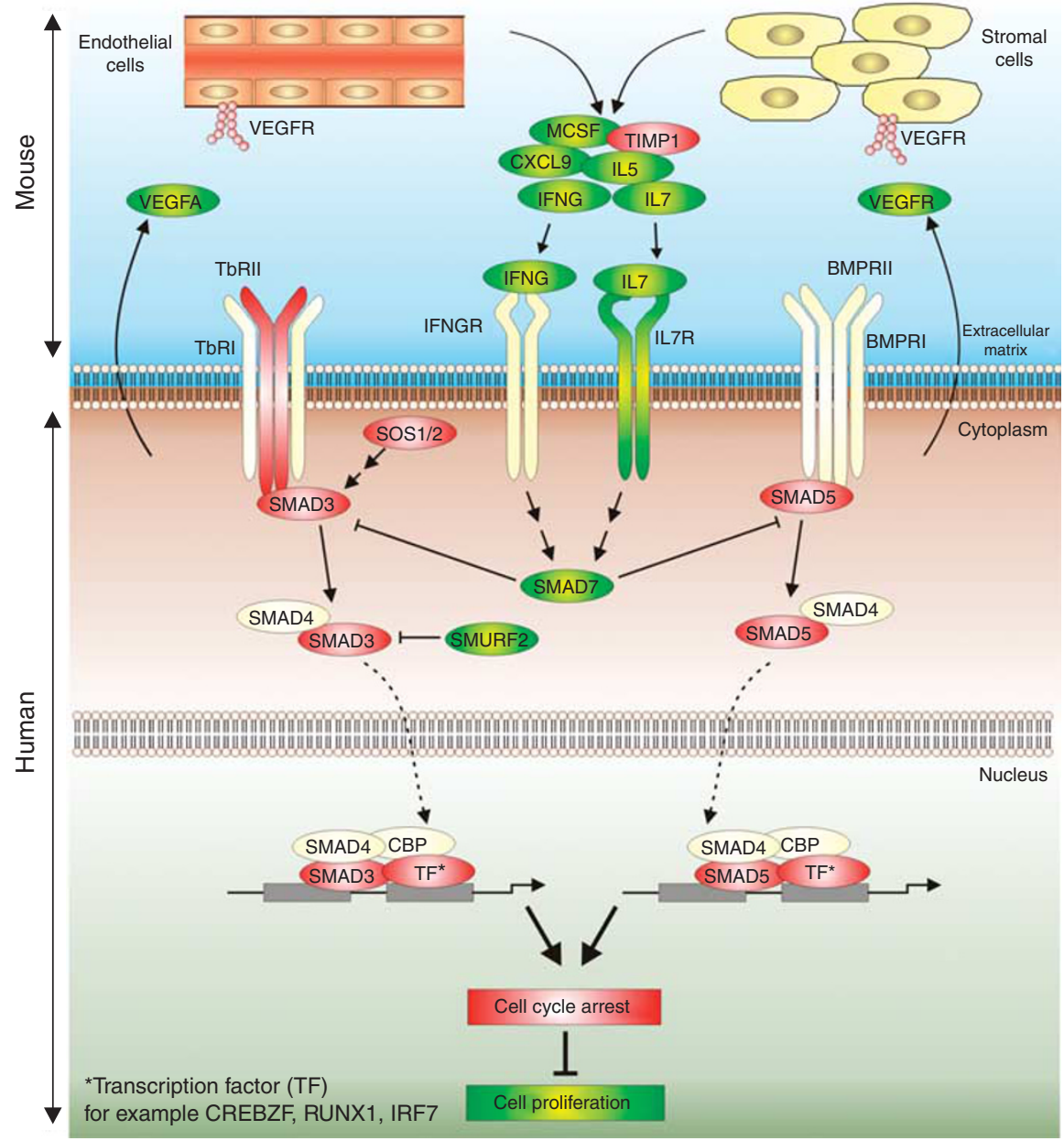

Figure 4 Proposed mechanism for VEGFAI 65 induced tumour growth. VEGFA production from the (human) tumour cells results in an increased fraction of host-derived stromal and endothelial cells. These (mouse) host-derived supportive cells produce a variety of cytokines that act on the (human) tumour cells. As a result, genes involved in the TGF- $\beta$ signalling pathway are differentially expressed, inducing the inhibition of TGF- $\beta$ signalling. This, in turn, results in decreased cell cycle arrest, which may explain the increased growth of VEGFA overexpression in tumour. The colours represent higher expressed (green) and lower expressed (red) genes or cytokines within the VEGFAI65 tumours; stimulatory effect; indirect stimulatory effect; -| inhibitory effect; - - > translocation.

Our data show that tumours overexpressing VEGFA165 were characterised by enhanced vessel outgrowth. An increase in MVD is seen in many malignancies, including haematological malignancies, with VEGFA as a key player (Carmeliet and Jain, 2000; Padro et al, 2000; de Bont et al, 2001). Vessels are known for their supply of oxygen and nutrients to the tumour cells, but also for 
excretion of various endothelial-derived growth factors of potential benefit for tumour cells (Fiedler et al, 1997; Dankbar et al, 2000). Our results show that endothelial and/or stromal cells within the tumour express higher levels of mouse-specific cytokines (MCSF, IFNG, IL5, IL7 and CXCL9) in VEGFA165 tumours compared with control tumours (Figure 4). From the literature it is known that exposure of human endothelial cells to VEGFA resulted in an increased production of cytokines, including MCSF (Fiedler et al, 1997; Bellamy et al, 1999; Dankbar et al, 2000). These results suggest that the effect of VEGFA165 is stromal dependent.

New is that within the human tumour cells genes involved in the TGF- $\beta$ signalling pathway were found to be differentially expressed between VEGFA165 tumours and control tumours. The TGF- $\beta$ signalling pathway is a negative regulator of cell cycle progression, resulting in a cell cycle arrest via transcription of TGF- $\beta$ responsive genes. TGFBR2, SMAD3, SMAD5, SOS1 and SOS2 were found to be lower expressed in VEGFA165 tumours compared with control tumours. In addition SMAD7 was found to be higher expressed in VEGFA165 tumours compared with control tumours. SMAD7 inhibits TGF- $\beta$ signalling by preventing the activation of other SMADs (Kleeff et al, 1999). These results suggest that the block in cell cycle progression is decreased via downregulation of TGF- $\beta$-responsive genes, resulting in cell proliferation. This is in line with our observation that VEGFA165 tumours showed an increased fraction of proliferating cells.

As no proliferative advantage was seen for the VEGFA165 cells in vitro we hypothesised that the TGF- $\beta$ signalling pathway is a result of the interaction with environment. Gene expression analysis revealed that nearly all of the genes involved in the TGF- $\beta$ signalling pathway were not differentially expressed between the VEGFA165 HL-60 cell lines and control HL-60 cell lines. Thus, our results are in concordance with the knowledge that stromal support is essential in tumour growth in vivo (Ayala et al, 2009).

Alterations in the TGF- $\beta$ signalling pathway are found in human malignancies, including haematological malignancies (Elliott and
Blobe, 2005; Dong and Blobe, 2006). Proposed mechanisms for the reduced TGF- $\beta$ signalling include a decrease in TGFBR2 expression or an increase in SMAD7 expression (Kim et al, 1999; Kleeff et al, 1999). In our study, tumours overexpressing VEGFA165 showed lower expression of TGFBR2 and higher expression of SMAD7, suggesting a resistance to the TGF- $\beta$-induced cell cycle arrest. The expression of SMAD7 can be regulated by various genes and cytokines, for example, IFNG and IL7 (Ulloa et al, 1999; Huang et al, 2002). Our data revealed that, in response to VEGFA expression by the tumour cells, these host-derived cytokines were upregulated. In addition, our microarray data showed that (human) IL7R was upregulated in the VEGFA165 tumours. Together, these data suggest that VEGFA165 induces the expression of host-derived growth factors and cytokines, which in turn induces inhibition of the TGF- $\beta$ signalling pathway (Figure 4).

In conclusion, we show that VEGFA165 significantly enhances tumour growth and tumour angiogenesis. We demonstrated that tumour-derived VEGFA resulted in enhanced tumour cell proliferation possibly by a paracrine inhibition of TGF- $\beta$ signalling within the tumour. These results indicate that the microenvironment is essential for VEGFA-induced tumour growth. Combining conventional therapeutic strategies with drugs targeting the tumour environment may be of benefit for tumour treatment.

\section{ACKNOWLEDGEMENTS}

We would like to thank Dr Bart-Jan Wierenga and Professor Dr Jan Jacob Schuringa for the kind gift of the retroviral construct pMSCV-iGF. This work was supported by a grant from the 'Dutch Cancer Society' to ESJM de Bont (3661).

Supplementary Information accompanies the paper on British Journal of Cancer website (http://www.nature.com/bjc)

\section{REFERENCES}

Aguayo A, Estey E, Kantarjian H, Mansouri T, Gidel C, Keating M, Giles F, Estrov Z, Barlogie B, Albitar M (1999) Cellular vascular endothelial growth factor is a predictor of outcome in patients with acute myeloid leukemia. Blood 94: 3717-3721

Ayala F, Dewar R, Kieran M, Kalluri R (2009) Contribution of bone microenvironment to leukemogenesis and leukemia progression. Leukemia 23: $2233-2241$

Bellamy WT, Richter L, Frutiger Y, Grogan TM (1999) Expression of vascular endothelial growth factor and its receptors in hematopoietic malignancies. Cancer Res 59: 728-733

Carmeliet P, Jain RK (2000) Angiogenesis in cancer and other diseases. Nature 407: 249-257

Dankbar B, Padro T, Leo R, Feldmann B, Kropff M, Mesters RM, Serve H, Berdel WE, Kienast J (2000) Vascular endothelial growth factor and interleukin-6 in paracrine tumor-stromal cell interactions in multiple myeloma. Blood 95: 2630-2636

de Bont ES, Fidler V, Meeuwsen T, Scherpen F, Hahlen K, Kamps WA (2002) Vascular endothelial growth factor secretion is an independent prognostic factor for relapse-free survival in pediatric acute myeloid leukemia patients. Clin Cancer Res 8: 2856-2861

de Bont ES, Rosati S, Jacobs S, Kamps WA, Vellenga E (2001) Increased bone marrow vascularization in patients with acute myeloid leukaemia: a possible role for vascular endothelial growth factor. Br J Haematol 113: 296-304

Dong M, Blobe GC (2006) Role of transforming growth factor-beta in hematologic malignancies. Blood 107: $4589-4596$

Elliott RL, Blobe GC (2005) Role of transforming growth factor Beta in human cancer. J Clin Oncol 23: 2078-2093

Fiedler W, Graeven U, Ergun S, Verago S, Kilic N, Stockschlader M, Hossfeld DK (1997) Vascular endothelial growth factor, a possible paracrine growth factor in human acute myeloid leukemia. Blood 89: 1870-1875
Grandage VL, Gale RE, Linch DC, Khwaja A (2005) PI3-kinase/Akt is constitutively active in primary acute myeloid leukaemia cells and regulates survival and chemoresistance via NF-kappaB, Mapkinase and p53 pathways. Leukemia 19: $586-594$

Hermani A, De Servi B, Medunjanin S, Tessier PA, Mayer D (2006) S100A8 and S100A9 activate MAP kinase and NF-kappaB signaling pathways and trigger translocation of RAGE in human prostate cancer cells. Exp Cell Res 312: $184-197$

Huang M, Sharma S, Zhu LX, Keane MP, Luo J, Zhang L, Burdick MD, Lin YQ, Dohadwala M, Gardner B, Batra RK, Strieter RM, Dubinett SM (2002) IL-7 inhibits fibroblast TGF-beta production and signaling in pulmonary fibrosis. J Clin Invest 109: $931-937$

Kim WS, Park C, Jung YS, Kim HS, Han J, Park CH, Kim K, Kim J, Shim YM, Park K (1999) Reduced transforming growth factor-beta type II receptor (TGF-beta RII) expression in adenocarcinoma of the lung. Anticancer Res 19: $301-306$

Kleeff J, Ishiwata T, Maruyama H, Friess H, Truong P, Buchler MW, Falb D, Korc M (1999) The TGF-beta signaling inhibitor Smad7 enhances tumorigenicity in pancreatic cancer. Oncogene 18: $5363-5372$

Lee CY, Tien HF, Hu CY, Chou WC, Lin LI (2007) Marrow angiogenesisassociated factors as prognostic biomarkers in patients with acute myelogenous leukaemia. Br J Cancer 97: 877-882

Lewis TS, Shapiro PS, Ahn NG (1998) Signal transduction through MAP kinase cascades. Adv Cancer Res 74: 49-139

Liu D, Jia H, Holmes DI, Stannard A, Zachary I (2003) Vascular endothelial growth factor-regulated gene expression in endothelial cells: KDRmediated induction of Egr3 and the related nuclear receptors Nur77, Nurr1, and Nor1. Arterioscler Thromb Vasc Biol 23: 2002-2007

Mani M, Carrasco DE, Zhang Y, Takada K, Gatt ME, Dutta-Simmons J, Ikeda H, Diaz-Griffero F, Pena-Cruz V, Bertagnolli M, Myeroff LL, 
Markowitz SD, Anderson KC, Carrasco DR (2009) BCL9 promotes tumor progression by conferring enhanced proliferative, metastatic, and angiogenic properties to cancer cells. Cancer Res 69: 7577-7586

Nicolas E, Ramus C, Berthier S, Arlotto M, Bouamrani A, Lefebvre C, Morel F, Garin J, Ifrah N, Berger F, Cahn JY, Mossuz P (2011) Expression of S100A8 in leukemic cells predicts poor survival in de novo AML patients. Leukemia 25: $57-65$

Padro T, Ruiz S, Bieker R, Burger H, Steins M, Kienast J, Buchner T, Berdel WE, Mesters RM (2000) Increased angiogenesis in the bone marrow of patients with acute myeloid leukemia. Blood 95: 2637-2644

Siapati EK, Papadaki M, Kozaou Z, Rouka E, Michali E, Savvidou I, Gogos D, Kyriakou D, Anagnostopoulos NI, Vassilopoulos G (2011) Proliferation and bone marrow engraftment of AML blasts is dependent on beta-catenin signalling. Br J Haematol 152: $164-174$

Tsujii M, Kawano S, Tsuji S, Sawaoka H, Hori M, Dubois RN (1998) Cyclooxygenase regulates angiogenesis induced by colon cancer cells. Cell 93: $705-716$

Ulloa L, Doody J, Massague J (1999) Inhibition of transforming growth factor-beta/SMAD signalling by the interferon-gamma/STAT pathway. Nature 397: $710-713$

Weidenaar AC, de Jonge HJ, Fidler V, Ter Elst A, Meeuwsen-de Boer T, Douwes J, Bouma-Ter Steege JC, Hahlen K, Kamps WA, de Bont ES (2008) Addition of PTK787/ZK 222584 can lower the dosage of amsacrine to achieve equal amounts of acute myeloid leukemia cell death. Anticancer Drugs 19: $45-54$

This work is published under the standard license to publish agreement. After 12 months the work will become freely available and the license terms will switch to a Creative Commons Attribution-NonCommercial-Share Alike 3.0 Unported License. 